Reprod. Nutr. Dévelop., 1982, 22 (1 A), 27-40.

\title{
Inefficacité d'une suralimentation imposée dès la naissance pour modifier le comportement alimentaire, la croissance et la composition corporelle ultérieurs du poulet
}

\author{
X. ALLAIN, J. SIMON (1) \\ Avec la collaboration technique de Bernadette CHEVALIER et M. DEROUET
}

Station de Recherches avicoles I.N.R.A. Nouzilly, 37380 Monnaie, France

Summary. Ineffectiveness of post-hatching overfeeding in modifying the subsequent appetite, weight gain and body composition of the chicken.

The effects of post-hatching overfeeding were studied in the chicken. Hubbard and Leghorn chickens were force-fed daily from 0 to 9 days of age by a factor of 1.5 times the food intake of the ad libitum-fed group, then 1.2 times that intake from 10 to 15 days. Thereafter, they were refed ad libitum. As might be expected, body weights were increased at the end of the forced-feeding period in overfed chickens of both breeds. Liver weight and malic enzyme activity and the size of adipocytes isolated from subcutaneous and gizzard adipose tissues (only measured in the Leghorn breed) were also increased by overfeeding. After ad libitum refeeding, weekly food intake and body weight gain up to 56 days of age were normal in overfed Hubbard chickens as compared to the controls. In overfed Leghorn chickens, those parameters decreased temporarily (1-2 weeks) and then became normal. At $\mathbf{2 8}$ and 35 days of age, the hourly distribution of daily food intake was not modified by prior overfeeding. Fasting plasma glucose levels were significantly lower at 15 and 42 days of age but normal at 56 days in overfed chickens. Glucose tolerance at 15, 42 and 56 days and abdominal fat content at 56 days were not modified by overfeeding. The glucose tolerance of Leghorn chicks was similar to that of Hubbard chickens at 14 days, but faster at 42 and 56 days. Therefore, overfeeding chickens immediately after hatching does not affect subsequent appetite, body weight gain or body fat content. This is contrary to the results observed in the rat and the mouse.

\section{Introduction.}

Dans certaines espèces comme l'Homme, le Rat, la Souris, l'alimentation imposée lors des premiers jours de la vie post-natale détermine la composition corporelle ultérieure. Certaines " obésités " apparaissent induites par une suralimentation pendant le jeune âge. Ainsi, de jeunes ratons ou souriceaux suralimentés pendant la période d'allaitement par diminution du nombre de petits sous la

(1) La correspondance doit être adressée à J. Simon. 
mère, puis sevrés et nourris ad libitum, présentent à long terme par rapport à ceux élevés en portée nombreuse ou "normale " un développement pondéral supérieur et une adiposité accrue (Widdowson et McCance, 1960; Knittle et Hirsch, 1968 ; Aubert, Suquet et Lemonnier, 1971 et 1980 ; Faust, Johnson et Hirsch, 1980). Cet engraissement exagéré résulte à la fois d'une hyperplasie et d'une hypertrophie des adipocytes (Knittle et Hirsch, 1968 ; Aubert, Suquet et Lemonnier, 1971, 1980). Enfin, la suralimentation pendant le jeune âge augmente la consommation alimentaire après sevrage au moins chez le Rat (Oscaï et McGarr, 1978).

Chez le Poulet, peu de travaux ont porté sur les répercussions du niveau de l'alimentation imposée pendant le jeune âge. Les plus développés concernent l'effet des restrictions sévères qui retardent de façon irréversible la croissance (Leclercq et al., 1970 ; March et Hensen, 1977 ; Ballam et March, 1979). Par contre, les effets d'une suralimentation n'ont été que peu étudiés. Seuls Nir et al. (1978) ont tenté de suralimenter des poussins de souche lourde ou légère dès l'âge de 3 jours pendant 18 jours. Les animaux sont alimentés ad libitum et, en outre, gavés deux fois par jour. En fait, la suralimentation (consommation spontanée + gavage) ne peut être imposée dès le départ : elle ne devient effective qu'au bout de 9 jours dans la souche légère et de 13 dans la souche lourde. Elle apparaît donc très brève ( 9 et 5 jours respectivement). Après 18 jours, il en résulte un engraissement et un poids corporel supérieurs chez les gavés. Mais, après alimentation libre, dans les deux souches, il n'y a pas d'augmentation de la consommation et, par ailleurs, les différences de poids vif et d'engraissement s'estompent à 9 semaines. On peut se demander si cette absence d'effets à long terme n'est pas la conséquence des conditions expérimentales qui ont conduit chez le poulet à une suralimentation " tardive " et brève.

Dans cette espèce, nous avons imposé dès la naissance à des poussins de souche lourde ou légère, une suralimentation à l'aide de gavages (150 puis 120 p. 100 de la consommation des témoins) et réexaminé les effets ultérieurs sur le comportement alimentaire et la composition corporelle. En outre, le poids du foie, l'activité de l'enzyme malique dans le foie et le diamètre des cellules adipeuses sont mesurés à la fin de la période de gavage.

\section{Matériel et méthodes.}

1. Animaux - Lots - Régimes. - A 1 jour, 130 poussins mâles de souche lourde (Hubbard, type Cornish $\times$ White Rock) et 130 poussins de souche légère (Leghorn) sont pesés et bagués. Dans chaque souche, trois lots homogènes pour le poids sont constitués. Un lot de 50 animaux " témoin » reçoit à volonté un régime de démarrage granulé dont la composition et les caractéristiques sont indiquées dans le tableau 1. Dans le deuxième lot (30 animaux), les poussins sont nourris par gavages avec le même aliment laissé sous forme de farine. Pour la fabrication de celui-ci, chaque ingrédient est broyé finement avant constitution du mélange et addition du complément minéral et vitaminique. Ce régime présente un caractère semi-synthétique dont l'utilisation est apparue indispensable lors 
d'essais préliminaires pour réussir la suralimentation dès l'éclosion. Pour contrôler l'existence éventuelle d'une inappétence ou d'une mauvaise utilisation liée à ce régime, un troisième lot de 50 animaux reçoit un aliment de démarrage du commerce, présenté sous forme de miettes, à base de maïs, blé, tourteau de soja. Les deux régimes ont permis des croissances similaires, les performances des animaux du $3^{\text {e }}$ lot ne sont donc pas présentées dans les résultats. L'eau est disponible en permanence. Les lots témoins sont alimentés à volonté de $7 \mathrm{~h}$ à $21 \mathrm{~h}$. Chaque lot est placé dans une cellule de $2 \times 2,40 \mathrm{~m}$, chauffée à l'aide d'une lampe à infrarouge. L'éclairement de $24 \mathrm{~h}$ les cinq premiers jours, passe à $16 \mathrm{~h}$ (de $7 \mathrm{~h}$ à $23 \mathrm{~h}$ ) jusqu'à deux semaines.

a) Période de gavage. Le gavage des poussins se fait par l'introduction dans le jabot d'un tuyau souple relié à une seringue graduée. L'aliment est mélangé

\section{TABLEAU 1}

Composition (p. 100) et caractéristiques des régimes démarrage et croissance

\begin{tabular}{|c|c|c|}
\hline & $\begin{array}{c}\text { Démarrage }(0-2 \mathrm{~s}) \\
(\text { p. } 100)\end{array}$ & $\begin{array}{c}\text { Croissance }(2-8 \mathrm{~s}) \\
\text { (p. } 100)\end{array}$ \\
\hline Maïs & 24,0 & 63,95 \\
\hline Amidon de maïs & 39,5 & - \\
\hline Gluten de maïs . & 7,0 & - \\
\hline Protéines de soja & 15,0 & - \\
\hline Tourteau de soja (50) & - & 26,0 \\
\hline Farine de Poisson (Norvège) $\ldots \ldots \ldots \ldots \ldots \ldots \ldots$ & 7,0 & 3,0 \\
\hline Huile $d^{\prime}$ arachide $\ldots \ldots \ldots \ldots \ldots \ldots \ldots \ldots \ldots \ldots$ & 2,5 & - \\
\hline Huile de maïs $\ldots \ldots \ldots \ldots \ldots \ldots \ldots$ & - & 3,0 \\
\hline DL-méthionine . & 0,14 & 0,1 \\
\hline L-tryptophane & 0,07 & - \\
\hline Carbonate de calcium & 1,0 & 1,5 \\
\hline Phosphate bicalcique . & 1,5 & 1,4 \\
\hline Sel iodé ............ & - & 0,3 \\
\hline Oligo-éléments (1) & 0,2 & 0,2 \\
\hline Mélange minéral (2) & 0,71 & - \\
\hline Mélange vitaminique $(3,4)$ & 1,33 & 0,5 \\
\hline Anticoccidien $"$ Pancoxin ${ }^{\prime} \ldots \ldots \ldots \ldots \ldots \ldots$ & 0,05 & - \\
\hline Anticoccidien " Stenorol " ...................... & - & 0,05 \\
\hline $\begin{array}{l}\text { Énergie métabolisable }(5) \text { (mesurée, } \mathrm{Kcal} / \mathrm{kg}) \ldots \ldots \ldots \\
\text { Énergie métabolisable }(\mathrm{calculée}, \mathrm{Kcal} / \mathrm{kg}) \ldots \ldots \ldots \ldots \\
\text { Matières azotées totales }(\mathrm{N} \times 6,25) \ldots \ldots \ldots \ldots \ldots\end{array}$ & $\begin{array}{l}3523 \\
3400 \\
25,0\end{array}$ & $\begin{array}{l}3 \overline{084} \\
19,8\end{array}$ \\
\hline
\end{tabular}

(1) Le mélange en oligo-éléments apporte (en g/kg du complément) : Cu 8,75; 1 1,285; Mn 110 ; Zn 100 ; Co 0,875 ; Fe 35 ; Se 0,150 .

(2) En g pour $100 \mathrm{~kg}: \mathrm{MgSO}_{4}, 7 \mathrm{H}_{20} 0,26 ; \mathrm{KH}_{2} \mathrm{PO}_{4} 0,35 ; \mathrm{Na}_{2} \mathrm{CO}_{3} 0,10$.

(3) Mélange vitaminique " démarrage ": Vit $A(500000 \mathrm{UI} / \mathrm{g}) 2,2$; Vit $\mathrm{D}_{3}(100000 \mathrm{UI} / \mathrm{g}) 3$; tocopherol (25 p. 100) 6 ; B.H.T. 12,5: ménadione 0,75 ; thiamine 0,2 ; riboflavine 0,600 ; pantothénate (Ca) 2 ; niacine 3 ; pyridoxine 0,$300 ; B_{12}(500 \mathrm{mg} / \mathrm{kg}) 0,160$; acide folique 0,15 ; biotine 0,02 ; choline (chlorure à $50 \%$ ) 250 ; support (maïs broyé) 839 .

$\left.{ }^{4}\right)$ Mélange vitaminique "croissance ": Vit A $(100000 \mathrm{Ul} / \mathrm{g}) 10$; Vit $D_{3}(100000 \mathrm{Ul} / \mathrm{g}) 1,5$; tocopherol $(25$ p. 100$) 6 ;$;.H.T. 12,5 ; ménadione 0,5 ; riboflavine 0,4 ; pantothénate $(\mathrm{Ca}) 0,8$; niacine 2,5 ; pyridoxine 0,1 ; Vit $\mathrm{B}_{12}(1 / 10000) 8$; acide folique 0,02 ; biotine 0,01 ; choline (chlorure à 25 p. 100) 200 ; support (avoine broyée) 258.

(5) Mesurée sur des coqs adultes. 
avant chaque gavage à de l'eau dans les proportions suivantes : eau 55 p. 100 , aliment $45 \mathrm{p} .100$. Compte tenu de sa densité, chaque $\mathrm{ml}$ de ce mélange apporte $0,5 \mathrm{~g}$ d'aliment sec. Auparavant, on enregistre la consommation des témoins, par pesée des mangeoires et contrôle du gaspillage, ce qui permet d'ajuster le niveau de suralimentation des animaux gavés. Les cinq premiers jours, 6 gavages sont effectués à $7,9,11,15,18$ et 21 h) puis, 5 les deux jours suivants $(7,10,15,18$ et $21 \mathrm{~h}$ ) et enfin 4 gavages à $7,11,15$ et $21 \mathrm{~h}$. La multiplication des gavages doit permettre de réussir la suralimentation et de minimiser la stimulation de la lipogenèse consécutive à la prise d'aliment par repas.

L'ingéré quotidien des "gavés " est fixé à 150 p. 100 des témoins les 9 premiers jours puis ramené à 120 p. 100 pour limiter une mortalité devenant importante $(5 / 30)$ chez les poulets Hubbard. Cette mortalité s'explique par un étouffement pendant le sommeil à la suite d'une régurgitation d'aliment obstruant la trachée aftère. En outre, certains poulets Leghorn gavés $(3 / 28)$ présentent un arrêt du transit digestif avec un jabot constamment distendu qui se traduit par un amaigrissement important. A l'âge de 16 jours, les gavages sont arrêtés de façon impromptue, le taux de mortalité atteignant à ce stade près de 50 p. 100 dans la souche Hubbard.

b) Période d'alimentation libre. A la fin de la période de gavage, 18 témoins et 18 gavés de chaque souche sont alors transférés dans une cellule dont la température et l'éclairement sont contrôlée (température décroissante de 28 à $20^{\circ} \mathrm{C}$ en fonction de l'âge ; $16 \mathrm{~h}$ d'éclairement de $7 \mathrm{~h}$ à $23 \mathrm{~h}$ ). Les témoins Hubbard et Leghorn (18/48) et gavés Leghorn (18/28) sont choisis en respectant les différentes classes de poids. Pour les gavés Hubbard, deux individus (léger et lourd) extrêmes sont éliminés. Les poulets sont alors élevés en cage individuelle et disposent à volonté d'eau et d'un aliment de croissance (granulé) dont la composition est indiquée dans le tableau 1. L'expérience initialement prévue jusqu'à 10 semaines est arrêtée à 8 semaines ; 2 poulets Leghorn (1 gavé et 1 témoin) présentent les symptômes de la maladie de Mareck.

\section{Mesures.}

a) Période de gavage. Les animaux sont pesés à jeun chaque semaine. A la fin du gavage, la taille des adipocytes est mesurée sur 8 Leghorn témoins et 8 gavés après une nuit de jeûne dans deux dépôts : sous la peau et autour du gésier. Simultanément, les foies sont prélevés, pesés, congelés dans l'azote liquide et conservés à $-20^{\circ} \mathrm{C}$ pour la mesure de l'activité de l'enzyme malique (10 poulets/lot).

b) Période d'alimentation libre. La consommation individuelle et le poids corporel sont relevés chaque semaine. A 4 et 5 semaines, on enregistre la consommation horaire de $7 \mathrm{~h}$ à $23 \mathrm{~h}$. A 2, 6 et 8 semaines, une tolérance au glucose est effectuée. A 8 semaines, après abattage des animaux, les foies sont pesés, les graisses abdominales sont prélevées et pesées pour mesurer l'engraissement (Delpech et Ricard, 1965). 


\section{Méthodes.}

a) Taille des adipocytes. Après abattage des animaux, des lambeaux de tissu adipeux du gésier ou sous-cutané situé au niveau de la cage thoracique et de l'abdomen sont prélevés. L'isolement des adipocytes se fait d'après la méthode de Rodbell (1964) : des morceaux de chaque tissu (environ $200 \mathrm{mg}$ ) sont incubés en présence de $2,5 \mathrm{ml}$ de tampon Krebs-Ringer bicarbonaté $\mathrm{pH} \mathrm{7,4}$ à $37^{\circ} \mathrm{C}$ renfermant de l'albumine bovine ( $2 \mathrm{p} .100)$ et de la collagénase (Sigma, 0,5 $\mathrm{mg} / \mathrm{ml}$ ). Les tubes sont gazés pendant $15 \mathrm{sec}$ avec un mélange de 95 p. $100 \mathrm{O}_{2}$ et 5 p. 100 $\mathrm{CO}_{2}$. Après une heure d'incubation avec agitation vigoureuse, les cellules sont séparées par filtration sur soie japonaise et lavées 3 fois. Pour une suspension cellulaire, 3 aliquotes de $50 \mu$ l sont déposés sur une lame. Sur chaque lame, quatre champs différents sont photographiés (Lavau et al., 1977). Le comptage et la mesure de la taille des cellules se fait par lecture du négatif sur un lecteur de microfilm $3 \mathrm{M}$ en déplaçant une échelle micrométrique sur l'écran. Les cellules d'un diamètre inférieur à $10 \mu \mathrm{m}$ ne sont pas prises en considération. Pour chaque individu et chaque tissu, les cellules sont regroupées par classe de $10 \mu \mathrm{m}$ (de 10 à $120 \mu \mathrm{m})$ et leur nombre (100-120) est exprimé en p. $100 \mathrm{du}$ total.

b) Dosage de l'enzyme malique. Les foies sont broyés froids dans deux volumes d'une solution glacée de saccharose $(0,25 \mathrm{M})$ au Potter. Un aliquot $(0,500 \mathrm{ml})$ est prélevé pour le dosage des protéines (Lowry et al., 1951). L'homogénat est centrifugé à $+4{ }^{\circ} \mathrm{C}$ pendant $20 \mathrm{~min}$ à $38000 \mathrm{~g}$. Le surnageant est conservé, le culot est réextrait avec un volume et centrifugé de la même façon. Les deux surnageants sont réunis, conservés au froid. L'activité de l'enzyme malique est mesurée le lendemain à $300 \mathrm{~nm}$ selon la méthode de Ochoa (1955) avec un tam-

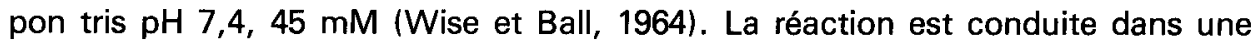
cuve thermostatée à $37^{\circ} \mathrm{C}$ en ajoutant successivement : 1,950 $\mathrm{ml}$ de tampon, $0,200 \mathrm{ml}$ de NADP $(0,75 \mathrm{mM}), 0,050 \mathrm{ml}$ d'échantillon, $0,100 \mathrm{ml}$ de $\mathrm{MnCl}_{2}$ (45 mM) puis $0,100 \mathrm{ml}$ de malate $(22 \mathrm{mM}, \mathrm{pH} 7,4)$. Pour éliminer les interférences avec le malate endogène, les échantillons sont incubés $4 \mathrm{~min}$ à la température de la pièce, puis équilibrés à $37^{\circ} \mathrm{C}$ (Tepperman et Tepperman, 1964). Les lectures sont effectuées toutes les $10 \mathrm{sec}$. L'activité est estimée par référence à un enzyme purifié à partir de foie de poulet (Sigma, $30 \mathrm{U} / \mathrm{mg}$ de protéines). Dans les conditions du dosage, la réponse est linéaire dans une zone de concentration allant de 0 à $16 \mu \mathrm{g}$.

c) Tolérance au glucose. A 2, 6 et 8 semaines, la tolérance au glucose est déterminée après une surcharge orale $(2 \mathrm{~g} / \mathrm{kg}$ de poids vif ; solution de glucose à $500 \mathrm{~g} / \mathrm{l}$ ). A 6 et 8 semaines, la mesure est effectuée le matin après un jeûne de $16 \mathrm{~h}$; à deux semaines, la période de suralimentation ayant été arrêtée brutalement, la tolérance au glucose est réalisée l'après-midi et le temps de jeûne varie : $5 \mathrm{~h}$ chez les témoins (après $2 \mathrm{~h}$ d'alimentation le matin), $17 \mathrm{~h}$ chez les gavés. A chaque âge, une prise de sang par animal est effectuée au niveau de la veine alaire à l'aide d'une seringue héparinée. Les prélèvements interviennent à 2 semaines aux temps : 0 (animaux ne recevant pas de glucose) et 30 min après la surcharge; à 6 semaines : 0 à $44 \mathrm{~min}$; enfin à 8 semaines : 0 et $60 \mathrm{~min}$. 
A chaque âge, les prélèvements après surcharge se situent après le pic de glycémie et d'insulinémie et les temps choisis correspondent au moment où des différences dans la vitesse de retour vers la glycémie basale (temps 0 ) se manifestent le mieux (Simon et Rosselin, 1979 ; Touchburn et al., 1981 ; Simon, résultats non publiés). Les échantillons sont placés à $0^{\circ} \mathrm{C}$ et centrifugés. Les plasmas sont prélevés, puis conservés à $-20^{\circ} \mathrm{C}$. La glycémie est mesurée par la méthode de la glucose oxydase à l'aide d'un analyseur Beckman et l'insulinémie par radioimmunologie en utilisant un sérum de cobaye anti-insuline de porc ( $A b$. 27-6, don du Dr. G. Rosselin, Hôpital Saint-Antoine, Paris) un traceur et un standard insuline de poulet (Simon et al., 1974 ; Simon et Rosselin, 1978).

d) Analyses statistiques. Les comparaisons entre traitements sont établies par test de $t$ et analyse de variance.

\section{Résultats.}

\section{Période de gavage.}

a) Consommation - Poids corporel. Après une semaine de suralimentation au niveau 150 p. 100 , les animaux gavés atteignent un poids corporel $(P<0,001)$ supérieur à celui des témoins : 22 p. 100 dans la souche Hubbard, 12 p. 100 dans la souche Leghorn (tabl. 2). Après 15 jours (le niveau de suralimentation étant ramené à 120 p. 100 à partir du dixième jour), les différences de poids restent significatives ( $P<0,001$, Leghorn ; $P<0,01$, Hubbard) mais ne sont plus que de 6 p. 100 dans les deux souches (tabl. 2). Chez les gavés la consommation cumulée est alors augmentée de 30 p. 100 (souche Hubbard) et 35 p. 100 (souche Leghorn).

TABLEAU 2

Poids vif ( $g$ ) et consommation alimentaire cumulée $(g)$ pendant la période de gavage

\begin{tabular}{|c|c|c|c|}
\hline Age (jours) & 0 & 7 & 15 \\
\hline \multicolumn{4}{|l|}{ Poids vif : } \\
\hline Hubbard - Témoins & $43,3 \underset{(50)}{ \pm} 0,4^{*}$ & $\underset{(50)}{88,3 \pm 1,5}$ & $\underset{(46)}{208 \pm 5}$ \\
\hline - Gavés & & $108,1_{(26)}^{ \pm} 1,3$ & $\underset{(20)}{221 \pm} 4$ \\
\hline Leghorn - Témoins & $36,8 \underset{(50)}{ \pm 0,2}$ & $\begin{array}{l}63,5 \pm 0,7 \\
(49)\end{array}$ & ${ }_{(49)}^{121 \pm 2}$ \\
\hline - Gavés & $37,1 \underset{(30)}{ \pm} 0,3$ & $\begin{array}{l}71,3 \pm 0,7 \\
(29)\end{array}$ & $\frac{127 \pm 2}{(28)}$ \\
\hline \multicolumn{4}{|l|}{ Consommation } \\
\hline Hubbard - Témoins & - & 53 & 244 \\
\hline - Gavés .. & - & 81 & 316 \\
\hline Leghorn - Témoins ... & - & 40 & 153 \\
\hline - Gavés $\ldots \ldots \ldots \ldots \ldots \ldots \ldots \ldots$ & - & 61 & 207 \\
\hline
\end{tabular}

* Moyenne \pm écart-type de la moyenne. Le nombre de poulets est indiqué entre parenthèses. 
b) Poids du foie et contenu en enzyme malique. Après 2 semaines de suralimentation, les différences de poids des foies (33 p. 100 d'augmentation chez les gavés) sont significatives $(P<0,01)$ (tabl. 3). Parallèlement, le contenu du foie en enzyme malique augmente, exprimé en valeur absolue ou par $\mathrm{mg}$ de protéines $(P<0,001)$.

\section{TABLEAU 3}

Poids $(g)$ et contenu en enzyme malique $(U)$ du foie à la fin de la période de gavage dans la souche Leghorn

\begin{tabular}{|c|c|c|c|}
\hline & $\begin{array}{l}\text { Poids frais } \\
\text { (g) }\end{array}$ & (U) ${ }^{E n z}$ & $\begin{array}{l}\text { malique } \\
\text { ( } \mathrm{mU} / \mathrm{mg} \text { protéines) }\end{array}$ \\
\hline $\begin{array}{l}\text { Témoins } \\
\text { Gavés . . }\end{array}$ & $\begin{array}{l}5,4 \pm 0,3^{*} \\
7,1 \pm 0,3\end{array}$ & $\begin{array}{l}129 \pm 12 \\
205 \pm 14\end{array}$ & $\begin{array}{l}100 \pm 7 \\
143 \pm 8\end{array}$ \\
\hline
\end{tabular}

* Moyenne \pm écart-type de la moyenne pour 10 poulets par lot.

c) Taille des adipocytes. Les histogrammes du diamètre des adipocytes dans les deux tissus adipeux sont présentés dans la figure 1. Hormis le cas du poulet 1 , les adipocytes ayant un diamètre compris entre 10 et $20 \mu \mathrm{m}$ sont en fréquence élevée dans les tissus adipeux du gésier et du sous-cutané des témoins. Par ailleurs, chez aucun poulet témoin, on observe des adipocytes ayant une taille supérieure à $90 \mu \mathrm{m}$. A l'inverse, chez les animaux gavés, même dans le cas du poulet 9 , pour lequel la classe $10-20 \mu \mathrm{m}$ reste importante, les classes de diamètre supérieur à $90 \mu \mathrm{m}$ jusqu'à $120 \mu \mathrm{m}$ sont présentes dans les deux tissus. En outre, les classes de diamètre supérieur à $60 \mu \mathrm{m}$ apparaissent nettement plus fréquentes. Le gavage a donc provoqué une hypertrophie importante des adipocytes.

\section{TABLEAU 4}

Consommation alimentaire cumulée $(g)$ et poids vif $(g)$ pendant la période d'alimentation libre

\begin{tabular}{|c|c|c|c|c|c|c|c|}
\hline Age (semaines) & & 3 & 4 & 5 & 6 & 7 & 8 \\
\hline 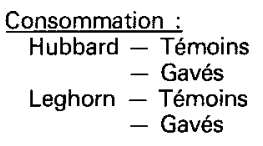 & $\begin{array}{l}(15) \\
(17) \\
(18) \\
(18)\end{array}$ & $\begin{array}{l}315 \pm 14^{*} \\
302 \pm 17 \\
146 \pm 4 \\
116 \pm 4\end{array}$ & $\begin{array}{l}794 \pm 29 \\
819 \pm 36 \\
388 \pm 7 \\
353 \pm 10\end{array}$ & $\begin{array}{r}1444 \pm 45 \\
1493 \pm 55 \\
657 \pm 11 \\
632 \pm 15\end{array}$ & $\begin{array}{r}2142 \pm 53 \\
2239 \pm 64 \\
963 \pm 22 \\
924 \pm 22\end{array}$ & $\begin{array}{l}2994 \pm 71 \\
3128 \pm 76 \\
1354 \pm 32 \\
1326 \pm 30\end{array}$ & $\begin{array}{l}3867 \pm 87 \\
4044 \pm 98 \\
1745 \pm 38 \\
1737 \pm 43\end{array}$ \\
\hline \multicolumn{8}{|l|}{ Poids vif: } \\
\hline $\begin{array}{c}\text { Hubbard } \\
- \text { Témoins } \\
\text { - Gavés } \\
\text { Leghorn }- \text { Témoins } \\
\text { - Gavés }\end{array}$ & $\begin{array}{l}(15) \\
(17) \\
(18) \\
(18)\end{array}$ & $\begin{array}{l}391 \pm 13 \\
396 \pm 17 \\
188 \pm 4 \\
174 \pm 3\end{array}$ & $\begin{array}{l}640 \pm 19 \\
661 \pm 30 \\
279 \pm 6 \\
266 \pm 7\end{array}$ & $\begin{array}{r}982 \pm 23 \\
1027 \pm 38 \\
386 \pm 9 \\
374 \pm 8\end{array}$ & $\begin{array}{r}1266 \pm 22 \\
1336 \pm 40 \\
484 \pm 12 \\
458 \pm 9\end{array}$ & $\begin{array}{r}1632 \pm 29 \\
1702 \pm 48 \\
601 \pm 14 \\
587 \pm 10\end{array}$ & $\begin{array}{r}1956 \pm 36 \\
2047 \pm 68 \\
726 \pm 20 \\
705 \pm 13\end{array}$ \\
\hline
\end{tabular}

* Moyenne \pm écart-type de la moyenne, le nombre de poulets étant indiqué entre parenthèses sur la même ligne. Dans la souche Leghorn, à 8 semaines, les moyennes de la consommation et du poids vif sont calculées sur 17 témoins et 16 gavés. 

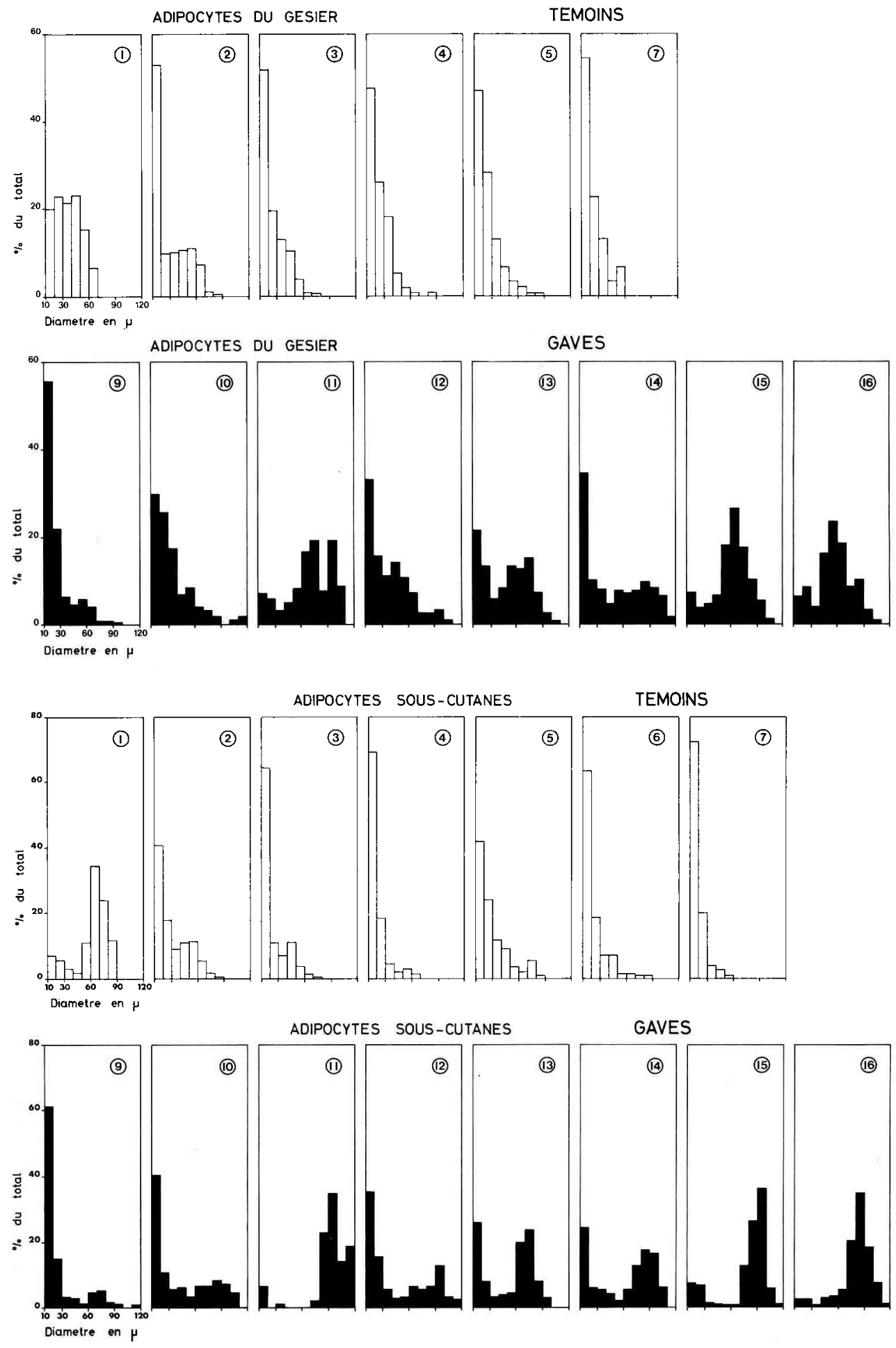


\section{Période d'alimentation libre.}

a) Consommation - Poids corporel. L'évolution de la consommation alimentaire cumulée pendant la période de réalimentation est présentée dans le tableau 4. Jusqu'à la fin de l'expérience, on n'observe pas de différences significatives entre Hubbard gavés et témoins. A l'inverse, la consommation des Leghorn gavés est significativement plus faible à 3 et 4 semaines $(P<0,01$, tabl. 4) par rapport aux témoins. En fait, la différence à 4 semaines résulte uniquement du retard de consommation accumulé pendant la première semaine d'alimentation libre. Par la suite, on n'observe plus de différence jusqu'à 8 semaines.

Après 6 jours de réalimentation libre ( 3 semaines d'âge), les différences de poids vif entre Hubbard gavés et témoins ne sont plus significatives (tabl. 4). Dans la souche Leghorn, le poids vif des gavés devient par contre significativement plus faible que celui des témoins $(P<0,05)$. Cette différence paraît reliée à la diminution de consommation observée chez les gavés dans cette souche pendant la première semaine de réalimentation. Par la suite, dans chaque souche, gavés et témoins présentent la même croissance.

A 4 semaines, l'étude de la prise alimentaire horaire au cours d'une journée ne révèle pas de différence liée au mode d'alimentation (fig. 2). Dans les deux souches, la consommation est importante pendant la première heure d'éclairement ; elle ehute à la deuxième et reste relativement stable jusqu'à la treizième où, au moins dans la souche Hubbard, on note une nette augmentation de l'ingéré horaire avant la baisse qui précède l'extinction de la lumière. Ces résultats sont confirmés à 5 semaines (données non présentées); les gavages n'ont donc pas modifié le comportement alimentaire.

TABLEAU 5

Poids $(g)$ du foie et des graisses abdominales à 8 semaines

\begin{tabular}{ccc}
\hline & Foie $(\mathrm{g})$ & Graisses abdominales $(\mathrm{g})$ \\
\hline Hubbard - Témoins (15) $\ldots \ldots \ldots \ldots$ & $36,9 \pm 1,4^{*}$ & $46,4 \pm 5,5$ \\
- Gavés (17) $\ldots \ldots \ldots \ldots$ & $37,5 \pm 1,4$ & $43,3 \pm 3,0$ \\
Leghorn - Témoins (17) $\ldots \ldots \ldots \ldots$ & $15,3 \pm 0,4$ & $3,1 \pm 0,7$ \\
- Gavés (16) $\ldots \ldots \ldots \ldots$ & $16,5 \pm 0,8$ & $3,0 \pm 0,6$ \\
\hline
\end{tabular}

* Moyenne \pm écart-type de la moyenne, le nombre de poulets étant indiqué entre parenthèses sur la même ligne.

A 8 semaines, l'engraissement des témoins et gavés, mesuré par le poids des graisses abdominales, est identique dans chaque souche (tabl. 5). Par ailleurs, les foies ne révèlent aucune différence de poids (tabl. 5), entre témoins et gavés.

FIG. 1. - Histogramme du diamètre des adipocytes des poulets Leghorn en fin de gavage. Parmi les 8 témoins prélevés, un ne présentait pas suffisamment de tissu adipeux sous-cutané et deux au niveau du gésier pour permettre une estimation de la taille des adipocytes. 

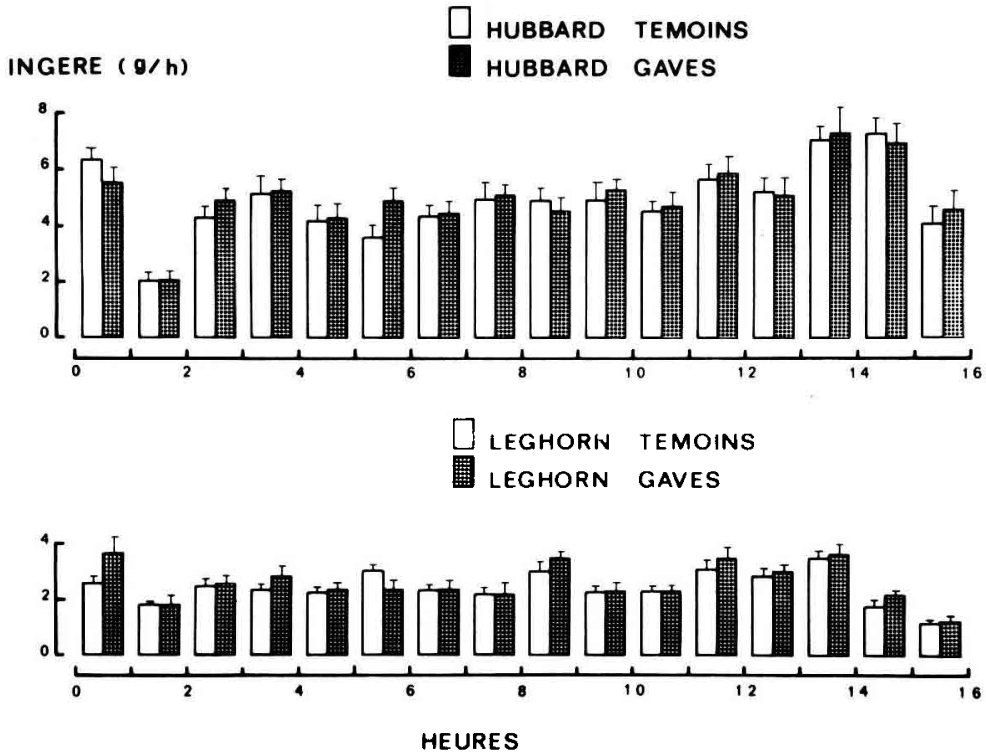

FIG. 2. - Évolution de la consommation horaire en fonction de l'heure d'éclairement à quatre semaines (moyenne +1 écart-type de la moyenne).

b) Glycémie - Insulinémie. A 2 et 6 semaines, la glycémie mesurée à jeun $\left(t_{0}\right)$ apparaît significativement plus faible chez les poulets gavés dans les deux souches $(P<0,01$, tabl. 6$)$. Les différences à 2 semaines ont pu être accusées par la durée variable du jeûne (17 h chez les gavés, $5 \mathrm{~h}$ chez les témoins). Néan-

TABLEAU 6

Tolérance au glucose en fin de gavage (15 jours) et pendant l'alimentation libre

\begin{tabular}{|c|c|c|c|c|c|c|}
\hline \multirow{2}{*}{$\begin{array}{l}\text { Age (jours) } \\
\text { Temps de prélèvement ( } \mathrm{min} \text { ) }\end{array}$} & \multicolumn{2}{|c|}{15} & \multicolumn{2}{|c|}{42} & \multicolumn{2}{|c|}{56} \\
\hline & 0 & 30 & 0 & 44 & 0 & 60 \\
\hline \multicolumn{7}{|l|}{ Glycémie $(\mathrm{mg} / 100 \mathrm{ml})$} \\
\hline $\begin{aligned} \text { Hubbard } & - \text { Témoins } \\
& - \text { Gavés } . . \\
\text { Leghorn } & - \text { Témoins } \\
& - \text { Gavés } \ldots\end{aligned}$ & $\begin{array}{l}199 \pm 10^{*} \\
178 \pm 4 \\
202 \pm 5 \\
178 \pm 5\end{array}$ & $\begin{array}{l}224 \pm 8 \\
214 \pm 6 \\
214 \pm 10 \\
218 \pm 6\end{array}$ & $\begin{array}{l}207 \pm 4 \\
194 \pm 5 \\
198 \pm 3 \\
187 \pm 2\end{array}$ & $\begin{array}{l}377 \pm 17 \\
327 \pm 18 \\
259 \pm 10 \\
235 \pm 7\end{array}$ & $\begin{array}{l}201 \pm 6 \\
191 \pm 4 \\
195 \pm 2 \\
189 \pm 4\end{array}$ & $\begin{array}{l}296 \pm 18 \\
277 \pm 10 \\
221 \pm 7 \\
229 \pm 12\end{array}$ \\
\hline
\end{tabular}

Insulinémie $(\mathrm{ng} / \mathrm{ml})$ :

\begin{tabular}{|c|c|c|c|}
\hline Hubbard - Témoins & - & - & $0,59 \pm 0,14 \quad 1,24 \pm 0,11 \quad 0,63 \pm 0,15 \quad 0,96 \pm 0,20$ \\
\hline - Gavés & - & - & $0,63 \pm 0,13 \quad 0,90 \pm 0,18 \quad 0,76 \pm 0,21 \quad 0,78 \pm 0,10$ \\
\hline Leghorn - Témoins & - & - & $1,16 \pm 0,19 \quad 0,37 \pm 0,04 \quad 0,87 \pm 0,17 \quad 0,62 \pm 0,17$ \\
\hline - Gavés & - & - & $1,42 \pm 0,39 \quad 0,29 \pm 0,05 \quad 1,69 \pm 0,41 \quad 0,51 \pm 0,14$ \\
\hline
\end{tabular}

* Moyenne \pm écart-type. Le nombre de poulets par groupe varie de 7 à 9 . A 42 jours, au temps 44, 2 poulets Hubbard témoins (insulinémie à 4,4 et $4,6 \mathrm{ng} / \mathrm{ml}$ ) et 1 Leghorn témoin (insulinémie à $3,72 \mathrm{ng} / \mathrm{ml}$ ) sont exclus de la moyenne. Il en est de même à 56 jours au temps 60 pour un Hubbard témoin (insulinémie à $6,24 \mathrm{ng} / \mathrm{ml}$ ). 
moins, la persistance d'une légère hypoglycémie à jeun chez les gavés à 6 semaines conduit à penser que les variations observées à 2 semaines ne résultent pas uniquement de différences dans la durée du jeûne. Par contre, l'effet des gavages disparaît à 8 semaines, les glycémies mesurées à jeun ne révèlent plus de différences significatives. Par ailleurs, entre souches, les glycémies basales sont semblables.

La tolérance au glucose n'a pas été modifiée par la suralimentation. En effet, les glycémies mesurées après surcharge (exprimées en valeur absolue ou par l'augmentation au-dessus du niveau initial) ne sont pas différentes entre témoins et gavés dans les deux souches à 2, 6 et 8 semaines. A 2 semaines, on n'observe pas non plus de différences entre Leghorn et Hubbard pour le retour vers la glycémie basale (glycémies identiques à $30 \mathrm{~min}$ ). A l'inverse, à 6 et 8 semaines, celui-ci est significativement $(P<0,01)$ plus rapide chez les Leghorn. Les insulinémies mesurées à jeun sont similaires entre témoins et gavés mais plus élevées chez les Leghorn ( $P<0,01$ à 6 semaines; $P<0,05$ à 8 semaines). Les prélèvements effectués après la surcharge de glucose interviennent après le pic de sécrétion d'insuline et ne peuvent renseigner que faiblement sur la quantité d'insuline secrétée. Après la surcharge en glucose, on n'observe pas de différences à 6 ou 8 semaines, consécutives à la suralimentation pendant le jeune âge. A l'inverse, les insulinémies sont plus faibles chez les poulets Leghorn $(P<0,05$ à 6 semaines : $F_{1,30}=3,94$ à 8 semaines, à la limite du seuil de signification). Dans cette souche, le retour vers la glycémie basale étant plus rapide, la sécrétion d'insuline est elle-même plus vite inhibée.

\section{Discussion. Conclusion.}

Après une semaine de suralimentation, les poussins gavés présentent des gains de poids supérieurs aux témoins. Mais à 15 jours, les différences sont moins importantes. Ce fléchissement résulte d'une diminution du niveau de suralimentation et probablement d'une synthèse accrue de lipides chez les gavés. L'existence d'une lipogenèse hépatique intense est effectivement suggérée, chez les Leghorn en fin de période de gavages, par l'augmentation notable du poids du foie, siège principal de la lipogenèse chez les oiseaux (Goodridge et Ball, 1967; O'Hea et Leveille, 1969) et son contenu en enzyme malique. Cet enzyme augmente dans le foie chez le poulet en cas d'hyperlipogenèse (Goodridge, 1968). Parallèlement, les lipides corporels sont accrus comme en témoignent l'augmentation du diamètre des adipocytes et l'observation des masses adipeuses à l'autopsie.

Après réalimentation libre, on n'observe pas de différences à long terme dans la consommation, la répartition de la prise alimentaire au cours de la journée, la croissance et la composition corporelle des poulets des deux souches. La validité de ces résultats ne peut être mise en cause par l'apparition de la maladie de Mareck dans la souche Leghorn à 7 semaines. En effet, cette maladie, qui classiquement est observée au début de la période de reproduction, se traduit par une chute brutale de l'appétit et une diminution du poids vif (Fritzsche et Gerriets, 1965). De telles manifesfations sont exclues dans le reste de l'effectif (tabl. 4). 
Contrairement à ce que l'on observe chez le rat (Oscaï et McGarr, 1978), il est impossible de stimuler à long terme la consommation alimentaire du poulet par une suralimentation forcée, imposée dès la naissance. La légère et transitoire inhibition de l'ingéré observée chez les Leghorn gavés pendant la première semaine de réalimentation peut refléter un ajustement plus précis de la prise alimentaire sur la composition corporelle (théorie lipostatique, Kennedy, 1953) ou un retour à une composition corporelle "normale " plus lent, inhérent aux caractéristiques de cette souche (maigre à croissance lente). En effet, dans la plupart des espèces telles que le poulet en croissance (Nir et al., 1974), le poulet adulte (Lepkovsky et Furuta, 1971), l'oie (Nir et al., 1973), le rat après sevrage (Cohn et Joseph, 1962), la prise d'aliment est inhibée lorsque l'adiposité a été préalablement élevée par une suralimentation forcée. La stimulation à long terme de la consommation chez le rat après alimentation pléthorique pendant la période d'allaitement (Oscaï et McGarr, 1978), constitue donc un cas particulier. A la naissance, la régulation de l'appétit apparaît encore modulable chez le rat mais serait fixée chez le poulet. Les variations possibles chez ce dernier touchent l'ajustement de la consommation spontanée en fonction de la qualité du régime.

Les seuls effets de la suralimentation observés concernent la légère hypoglycémie mesurée à jeun à 2 et 6 semaines. Cette différence est transitoire et n'apparaît pas associée à 6 semaines, dans les conditions de la mesure, à une sécrétion d'insuline plus élevée ou à une sensibilité accrue des tissus (il n'y a pas de baisse plus rapide de la glycémie chez les gavés). Par ailleurs, dans les deux souches, la glycémie après une surcharge de glucose diminue à la même vitesse à 2 semaines. A l'inverse, à 6 et 8 semaines, le retour vers la glycémie basale est plus rapide chez les poulets Leghorn. Cette opposition entre souches, confirme des résultats antérieurs (Hara et Simon, résultats non publiés). Elle peut refléter par analogie à la dégradation de la tolérance au glucose observée en cas d'obésité chez les mammifères, les différences de composition corporelle entre les deux souches (Hubbard : gras ; Leghorn : maigres). Enfin, contrairement à des résultats antérieurs (Hara et Simon, résultats non publiés), les poulets Leghorn présentent une insulinémie basale supérieure à celle des Hubbards. Cette augmentation qui n'est pas associée à une hypoglycémie, suggérerait une résistance tissulaire à l'insuline dans cette souche. Cela n'apparaît pas puisque la tolérance au glucose reste accélérée. Elle représenterait plutôt une réaction de l'organisme, liée à l'état infectieux (maladie de Mareck latente dans cette souche) modifiant soit le contrôle nerveux de la sécrétion d'insuline, soit l'équilibre endocrinien à l'état basal. On sait, en effet, qu'un excès de corticoïdes élève l'insulinémie basale du rat (Perley et Kipnis, 1966) et du hamster (Campbell et al., 1966).

Reçu en novembre 1980. Accepté en juillet 1981.

Remerciements. - Nous remercions $\mathrm{Cl}$. Bouchot pour le traitement des films photographiques. 


\section{Références}

AUBERT R., SUQUET J. P., LEMONNIER D., 1971. Effets à long terme de trois niveaux d'ingesta lactés sur les lipides corporels, la taille et le nombre de cellules adipeuses de la souris. C. R. Acad. Sci. Paris, Sér. D., 273, 2636-2638.

AUBERT C. R., SUQUET J. P., LEMONNIER D., 1980. Long term morphological and metabolic effects of early under- and over-nutrition in mice. J. Nutr., 110, 649-661.

BALLAM C. G., MARCH B. E., 1979. Adipocytes size and number in mature broiler type female chickens subjected to dietary restriction during the growing period. Poult. Sci., 58, 940-948.

CAMPBELL J., RASTOGI K. S., HAUSLER H. R., 1966. Hyperinsulinemia with diabetes induced by cortisone, and the influence of growth hormone in the chinese hamster. Endocrinology, 79, 749-756.

COHN C., JOSEPH D., 1962. Influence of body weight and body fat on appetite of " normal " lean and obese rats. Yale J. Biol. Med., 34, 598-607.

DELPECH P., RICARD F. H., 1965. Relation entre les dépôts adipeux viscéraux et les lipides corporels chez le poulet. Ann. Zootech., 14, 181-189.

FAUST I. M., JOHNSON P. R., HIRSCH J., 1980. Long-term effects of early nutritional experience on the development of obesity in the rat. J. Nutr. 110, 2027-2034.

FRITZSCHE K., GERRIETS E., 1965. Maladie des volailles, $2^{\circ}$ édit., Vigot Frères, Paris, 211-221.

GOODRIDGE A. G., 1968. Citrate-clivage enzyme, "Malic Enzyme " and certain deshydrogenases in embryonic and growing chicks. Biochem. J., 108, 663-666.

GOODRIDGE A. G., BALL E. G., 1967. Lipogenesis in the pigeon : in vivo studies. Am. J. Physiol., 213, 245-249.

KENNEDY G. C., 1953. The role of depot fat in the hypothalamic control of food intake in the rat. Proc. roy. Soc. London, Ser. B, 140, 578-592.

KNITTLE J. L., HIRSCH J., 1968. Effect of early nutrition on the development of rat epididymal fat pads : cellularity and metabolism. J. clin. Invest., 47, 2091-2098.

LAVAU M., SUSINI C., KNITTLE J., BLANCHET-HIRST S., GREENWOOD M. R. C., 1977. A reliable photomicrographic method for determining fat cell size and number : application to dietary obesity. Proc. Soc. exp. Biol. Med., 156, 251-256.

LECLERCQ B., SIMON J., BLUM J. C., CALET C., 1970. Influence des restrictions alimentaires intervenant dès la naissance sur les performances de ponte de deux souches de poulettes. Ann. Zootech., 19, 333-346.

LEPKOVSKY S., FURUTA F., 1971. The role of homeostasis in adipose tissues upon the regulation of food intake of White Leghorn cockerels. Poult. Sci., 51, 573-577.

LOWRY O. H., ROSEBROUGH N. J., FARR A. L., RANDALL R. J., 1951. Protein measurement with the folin phenol reagent. J. biol. Chem., 193, 265-275.

MARCH B. E., HENSEN G., 1977. Lipid accumulation and cell multiplication in adipose bodies in White Leghorn and broiler-type chicks. Poult. Sci., 56, 886-894.

NIR I., NITSAN Z., VAX A., 1973. The influence of force feeding and of protein supplementation to the diet on the metabolisable energy of diets, digestibility of nutrients, nitrogen retention and digestive enzymes output in geese. Ann. Biol. anim. Bioch. Biophys., 13, 465-479.

NIR I., SHAPIRA N., NITSAN Z., DROR Y., 1974. Force feeding effects on growth, carcass and blood composition in the young chick. Br. J. Nutr., 32, 229-239.

NIR I., NITSAN Z., DROR Y., SHAPIRA N., 1978. Influence of overfeeding on growth, obesity and intestinal tract in young chicks of light and heavy breeds. Br. J. Nutr., 39, 27-35.

OCHOA S., 1955. Malic Enzyme, 739-753. In COLOWICK S. P., KAPLAN N. O., Methods in enzymology, vol. I, Acad. Press, New York.

O'HEA E. K., LEVEILLE G. A., 1969. Lipid biosynthesis and transport in the domestic chick /Gallus domesticus). Comp. Biochem. Physiol., 30, 149-159.

OSCAİ L. B., McGARR J. A., 1978. Evidence that the amount of food consumed in early life fixes appetite in the rat. Am. J. Physiol., 235, R 141-R 144.

PERLEY M., KIPNIS D. M., 1966. Effect of glucocorticoïds on plasma insulin. N. Engl. J. Med., 274, 1237-1241. 
RODBELL M., 1964. Localisation of lipoprotein lipase in fat cells of rat adipose tissue.J. biol. Chem., 239, 753-755.

SIMON J., FREYCHET P., ROSSELIN G., 1974. Chicken insulin : radio-immunological characterisation and enhanced activity in rat fat cells and liver plasma membranes. Endocrinology, 95. 1439-1449.

SIMON J., ROSSELIN G., 1978. Effect of fasting, glucose, amino acids and food intake on in vivo insulin release in the chicken. Horm. Metab. Res., 10, 93-98.

SIMON J., ROSSELIN G., 1979. Effect of intermittent feeding on glucose-insulin relationship in the chicken. J. Nutr., 109, 631-641.

TEPPERMAN H. M., TEPPERMAN J., 1964. Patterns of dietary and hormonal induction of certain NADP-linked liver enzymes. Am. J. Physiol., 206, 357-361.

TOUCHBURN S. P., SIMON J., LECLERCO B., 1981. Evidence of a glucose-insulin imbalance and effect of dieteray protein and energy level in chickens selected for high abdominal fat content. J. Nutr., 111, 325-335.

WISE E. M., BALL E. G., 1964. Malic enzyme and lipogenesis. Proc. nat. Acad. Sci. USA, 52, 12551262.

WIDDOWSON E. M., McCANCE R. A., 1960. Some effects of accelerating growth. I. General somatic development. Proc. roy. Soc. London, 152, 188-206. 